\title{
Evaluation Measures for Adaptive PLI Filters in ECG Signal Processing
}

\author{
FC Chang ${ }^{1}$, CK Chang ${ }^{2}, \mathrm{KY} \mathrm{Chi}{ }^{2}, \mathrm{YD} \mathrm{Lin}{ }^{2}$ \\ ${ }^{1}$ Graduate Institute of Electrical and Communications Engineering, Feng Chia University, Taichung, \\ Taiwan \\ ${ }^{2}$ Department of Automatic Control Engineering, Feng Chia University, Taichung, Taiwan
}

\begin{abstract}
Many studies have been devoted to the adaptive power-line interference (PLI) filter design for ECG signal processing. However, almost all existing PLI suppression filters are developed for applications in which the presence of PLI noise is assumed a priori. Indiscriminate application of PLI suppression over an ECG signal that is free of PLI noise may deform ECG morphology, and even cause degraded performance of subsequent processing. To date, little work has been done on the possibility of ECG signal degradation by such filtering operation and the impact on further processing. In order to evaluate the difference between the original and the filtered pattern, this study proposes quantitative evaluation measures. The assessments include convergence time, the frequency tracking efficiency, the execution time and the relative statistics in time and frequency domain. Extensive experiments have been done with artificially and practically corrupted ECG signals for four existing PLI adaptive filtering techniques (Ahlstrom and Tompkins', Pei and Tseng's, So's and Ziarani and Konrad's algorithm). The results reveal that $E C G$ signal distortion resulted from these existing adaptive filters. None of the existing algorithms outperform the others in all assessments. The proposed evaluation measures can also be used for the performance evaluation of the other types of artifact suppression, such as the baseline wander and EMG corruption contaminated in ECG, after minor modification. The proposed measures also make the optimal filter design under different constraints possible for ECG signal processing.
\end{abstract}

\section{Introduction}

Power-line interference (PLI) may be the most popular interference during electrocardiogram (ECG). As power-line frequency may deviate from nominal 50 (or 60) $\mathrm{Hz}$, an adaptive filter which can track the statistical characteristics of reference signal by iteratively updating filter parameters to improve the filter efficacy is feasible to serve the purpose of PLI removal. Many studies have been published regarding adaptive PLI filter design for ECG signal processing [1-5]. For example, Ahlstrom and Tompkins proposed an adaptive algorithm that can estimate how much the sinusoidal PLI is present and subtract it from the corrupted signal, thus leaving only ECG portion of signal [2]. Pei and Tseng presented a $2^{\text {nd }}-$ order adaptive IIR notch filter based on the least mean $p$ power (LMP) error criterion [3]. In So's algorithm, the input signal is assumed to be modeled by the summation of the desired signal and sinusoidal interference with unknown amplitude and phase. By the steepest descent method, the iterative formulae for amplitude and phase can thus be derived [4]. Ziarani and Konrad's algorithm can trace the variation of the signal by core units by dealing with its fundamental and its harmonic components to remove the contaminated PLI buried in the input signals [5].

However, little research has been done on the performance evaluation and performance comparison for adaptive PLI filter in ECG signal processing. To attain this goal, this paper proposes evaluation measures in both time and frequency domain to evaluate the performance of filters for such purpose. These quantifiable measures include the time needed for numerical convergence, the efficacy of PLI frequency tracking, execution time and the relative RMS statistics in time-and frequency-domain operation. The algorithms mentioned above (Ahlstrom and Tompkins', Pei and Tseng's, So's and Ziarani and Konrad's algorithm) are included for tests. The proposed evaluation methods may facilitate the design of optimal PLI filter for ECG signal processing.

\section{Methods}

\subsection{Subject and device}

The ECG signals used in this study were obtained from two resources. One was those 
acquired from the MIT-BIH Arrhythmia Database (sampling frequency $360 \mathrm{~Hz}$ ). The others were obtained in a university laboratory for electronic experiments. For the latter case, Lead II ECG signals were collected from 10 male subjects aged 20-25 with MP30 (Biopac system Inc., sampling frequency $500 \mathrm{~Hz}$ ). Two procedures were conducted for ECG measurement. During the procedure of clean data measurement, power cord were positioned at least 1 meter away from the subject and all of the electronic equipment in the lab were turned off to make sure the ECG signal was not contaminated by PLI. To obtain ECG signals that were contaminated PLI, we used a desk lamp as a noise source and put a power line across the head of the subject. All of measured ECG signals have been checked via spectrum analysis to verify and ensure the classification.

\subsection{Data analysis}

The following assessments were used for evaluation measures in this study:

1) The time needed for numerical convergence: This term is used to evaluate the time consumption needed for the PLI removal to a specific level under the controlled condition that the simulated PLI with known characteristics is artificially added to a prescreened pure ECG signal. The needed times are estimated as the time consumption on which the following equation equals $1 \%$, $5 \%$ and $10 \%$, respectively.

$\frac{\text { Amplitude of residual PLI }}{\text { Amplitude of the added PLI }} \times 100 \%$

2) The efficacy of PLI frequency tracking: Regarding the efficiency of handling the PLI with slowly change frequency, the following two equations were used to simulate the PLI with in this experiment. One is

$$
\begin{aligned}
& x(t)=A \cdot \sin (2 \cdot \pi \cdot f \cdot t), \text { with } f=59+0.02 \cdot t, \\
& \text { and the other one is: } \\
& x(t)=A \cdot\left[\sin (2 \cdot \pi \cdot f \cdot t)+\frac{1}{3} \sin (2 \cdot \pi \cdot f \cdot t)\right], \\
& \text { with } f=59+0.02 \cdot t
\end{aligned}
$$

The former equation is used to simulate the PLI with a slowly increasing frequency, whereas the latter one is for the cases with harmonic component in addition to the changing frequency and can be applied to observe whether the algorithm can track the PLI with harmonic component.

3) The execution time: The execution time for each algorithm can be shown by Matlab ${ }^{\circledR}$ command, profile (with $\mathrm{AMD}^{\circledR}$ sempron $2600+, 2.13 \mathrm{G} \mathrm{Hz}$ clock and 512 MB RAM).

4) The relative RMS statistics in time- and frequencydomain operation: In time-domain operation, three different RMS values, root mean square deviation
(RMSD), root mean square error (RMSE) and root mean square variation (RMSV) are used to assess the filtering efficacy and the degree of distortion of the signal after running the algorithms.

RMSD is the RMS value obtained from the pure ECG signal minus the restored ECG signal that has been processed by the adaptive filter. A smaller RMSD value indicates a better efficacy of the adaptive filter in eliminating PLI and less distortion of signal after the filtering process.

RMSE is the RMS value of the difference between the restored ECG and the filter output for clean ECG. A smaller RMSE value indicates a lesser distortion of ECG morphology after the filtering operation. RMSE looks similar to RMSD, yet RMSE takes more considerations on the possibility of the ECG morphology distortion after filtering.

RMSV is the RMS value of the tiny variation from the original input ECG after it has been blindly processed by the algorithms. The RMSV indicates the degree of variation of the ECG signal processed by the adaptive filter. The flow chart for the three RMS values is shown in Figure 1.

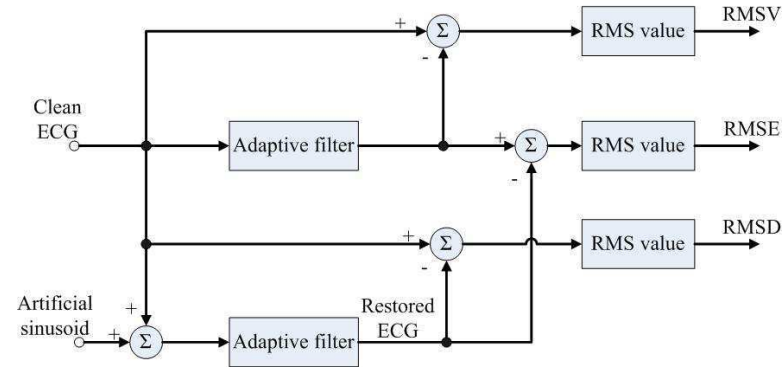

Figure 1 Flow chart for time-domain measures.

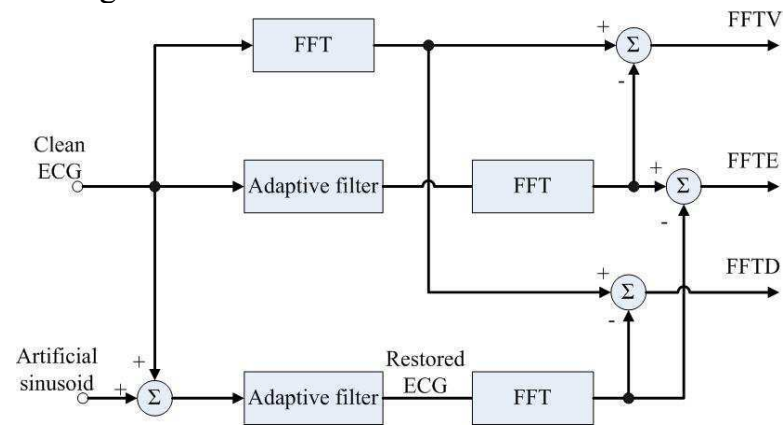

Figure 2 Flow chart for frequency-domain measures.

In addition, with the same philosophy as the assessment of RMS values in time domain, the frequency-domain assessments can also be attained as depicted in Figure 2. The corresponding RMS values for the amplitude and phase of FFTV, FFTE and FFTD are then derived to evaluate the possible distortion between the derived spectra.

All of the clean ECG signals selected from MIT-BIH 
arrhythmia database are adopted for this experiment. The statistics (means and standard deviation) for all of the evaluation measures are then derived for performance comparisons.

\section{Results}

\subsection{The time needed for numerical convergence}

The filtering results of PLI filters for the $60-\mathrm{Hz}$ sine wave with fixed amplitude. It can be appreciated that the convergence time of Ahlstrom and Tompkins' method and that of Pei and Tseng's method were the shortest for the case of convergence to $1 \%$. The convergence time of So's method was the longest and the output of the algorithm could not converge to $1 \%$. In addition, Ziarani and Konrad's method also could not converge to $1 \%$ but the time taken to converge to $5 \%$ and $10 \%$ was less than in So's method which took about 2 seconds and 1.5 seconds respectively.

\subsection{The efficiency of PLI frequency tracking}

As described in the section "Materials and methods", equation (2) and (3) were developed to simulate the PLI with slowly changing frequency and with harmonic component. The filtering results for a simulated PLI as defined in equation (2). It is easily appreciated that there lacks the capability of tracking PLI frequency for Ahlstrom and Tompkins' and So's algorithm. In So's algorithm, the frequency of PLI should be known and fixed a priori [4]. Besides, there has been reported that the Ahlstrom and Tompkins' algorithm is in fact an equivalent fixed notch filter [6].

Only Ziarani and Konrad's method has the ability to cancel the PLI with harmonic component because the specific core units for tackling fundamental and harmonic components can be arranged in parallel to serve the purpose [5].

\subsection{The execution time}

The execution time may imply the computational complexity of the algorithm. A clean ECG signal selected from MIT-BIH arrhythmia database (No. 112) with added simulated PLI (60 Hz sinusoid with amplitude of $1.5 \mathrm{mV}$ ) was used as the input signal in this experiment. By using the command profile in Matlab ${ }^{\circledR}$ the execution time of each algorithm can be extracted. In order to assess the relationship between data length and execution time, signal lengths of 60 and 180 seconds have been used for the test (as shown in Table1). Pei and Tseng's method has the shortest time consumption to reach $5 \%$ convergence. However, the execution speed of Ahlstrom and Tompkins', Pei and Tseng's and So's method increase remarkably as the input signal length was tripled.

\subsection{The relative RMS statistics in time- and frequency-domain operation.}

The relative RMS statistics are derived according to the processing flow as demonstrated in Figure 1 (for time-domain operation) and Figure 2 (for frequencydomain operation).

Experimental results (summarized in Table 1 and Table 2) demonstrate that Ahlstrom and Tompkins' method has the best behavior in RMSD, FFTD RMSE and FFTE when handling signals contaminated with simulated PLI. It means the algorithm has the best noise cancellation efficacy and the least ECG morphology distortion in both time- and frequency-domain operation. On the other hand, the Ziarani and Konrad's method has the best performance in RMSV which implies the smallest ECG pattern distortion may be resulted in as a clean ECG being blindly processed. However, these assessments only are under the simulated challenges. The quantifiable assessment results would be different in a normal environment. By using the ECG signals contaminated with PLI from a real power line as the input of the algorithms, only the RMSV and FFTV can be estimated since artificial sinusoid does not exist then. Ahlstrom and Tompkins' method does not perform remarkably in such a situation as compared with the simulation circumstance because of its weak frequency tracking efficiency. The Ziarani and Konrad's method demonstrates the best efficiency to eliminate PLI in a practical environment.

\subsection{Discussion}

Advantages and disadvantages of these four algorithms have been evaluated with extensive experiments. Regarding convergence time and frequency tracking, Pei and Tseng's method exhibits excellent performance even for PLI with slowly increasing frequency. For frequency tracking efficiency, the disadvantage of So's method and Ahlstrom and Tompkins' method was that the frequency cannot be updated iteratively. The main reason for the bad performance of So's method was that the PLI frequency should be set as a constant in the algorithm and only the amplitude and phase response are updated. Ahlstrom and Tompkins' algorithm in its essence is a notch filter [6] such that it is not able to trace the frequency.

All of this assessment results are summarized comprehensively in Table 3. It can be appreciated that none of the existing algorithms outperform the others in 
all assessments. To be noted, if the time consumption is critical for long-term ECG processing, Ziarani and Konrad's algorithm would be a better choice. In addition, Ziarani and Konrad's method is the only one capable of removing serve PLI with harmonic component among the four methods.

Table 1 Relative RMS statistics for simulation PLI.

\begin{tabular}{|c|c|c|c|c|}
\hline & Ahl & Pei & So & Kon \\
\hline \multicolumn{5}{|c|}{ Time domain $(\mathrm{mV})$} \\
\hline \multicolumn{5}{|l|}{ Mean } \\
\hline RMSD & $* 2.1 \pm 1.4$ & $13.7 \pm 10$ & $5.21 \pm 2.7$ & $8.09 \pm 4.8$ \\
\hline RMSE & $* 1.0 \pm 0.9$ & $28.9 \pm 21$ & $5.23 \pm 3.5$ & $8.14 \pm 4.9$ \\
\hline RMSV & $2.0 \pm 1.4$ & $24.1 \pm 15$ & $4.8 \pm 2.5$ & $0.75 \pm 0.6$ \\
\hline \multicolumn{5}{|c|}{ Frequency domain } \\
\hline \multicolumn{5}{|c|}{ Energy variation $\left(\mathrm{mV}_{\mathrm{rms}}\right)$} \\
\hline FFTD & $* 0.21 \pm 0.23$ & $35.3 \pm 5.4$ & $7.96 \pm 0.1$ & $0.27 \pm 0.6$ \\
\hline FFTE & $* 0.01 \pm 0.01$ & $20.9 \pm 10$ & $0.41 \pm 0.1$ & $0.23 \pm 0.5$ \\
\hline FFTV & $0.21 \pm 0.23$ & $16.1 \pm 8.8$ & $7.54 \pm 0.1$ & $* 0.01 \pm 0$ \\
\hline
\end{tabular}

Table 2 Relative RMS statistics for practical PLI.

\begin{tabular}{|c|c|c|c|c|}
\hline Time do & $\begin{array}{r}\text { Ahl } \\
\text { in }(\mathrm{mV})\end{array}$ & Pei & So & Kon \\
\hline Mean & & & \multirow{4}{*}{$* 30 \pm 27$} & \multirow{4}{*}{$32.4 \pm 35$} \\
\hline RMSV & $35.6 \pm 35$ & $54.6 \pm 21$ & & \\
\hline \multicolumn{3}{|c|}{ Frequency domain $\left(\mathrm{mV}_{\mathrm{rms}}\right)$} & & \\
\hline \multicolumn{3}{|c|}{ Energy varuation } & & \\
\hline FFTV & $0.19 \pm 0.1$ & $16.6 \pm 4.1$ & $7.26 \pm 0.2$ & $* 0.02 \pm 0$ \\
\hline
\end{tabular}

Table 3 Comprehensive assessments

\begin{tabular}{|c|c|c|c|c|}
\hline & Ahl & Pei & So & Kon \\
\hline $\begin{array}{l}\text { Convergence } \\
\text { time (sec.) }\end{array}$ & 0.32 & $* 0.1$ & 2.52 & 0.56 \\
\hline $\begin{array}{l}\text { Frequency } \\
\text { tracking }\end{array}$ & No & Yes & No & Yes \\
\hline $\begin{array}{l}\text { Harmonic } \\
\text { cancellation }\end{array}$ & No & No & No & Yes \\
\hline \multicolumn{5}{|c|}{ Computational time (sec) } \\
\hline $\begin{array}{l}\text { Short term } \\
(60 \mathrm{sec})\end{array}$ & 10.51 & $* 9.43$ & 10.62 & 24.12 \\
\hline $\begin{array}{l}\text { Long term } \\
(180 \mathrm{sec})\end{array}$ & 127.34 & 130.73 & 125.76 & *70.85 \\
\hline
\end{tabular}

Legend:

Ahl denote Ahlstrom and Tompkins' method

Pei denote Pei and Tseng's method

So denote So's method

Kon denote Ziarani and Konrad's method

* indicates the smallest among the results.

\section{Discussion and conclusions}

This study has presented quantitative evaluation measures for the performance assessments of the adaptive PLI filter in ECG signal processing. The results demonstrated that there is no universal optimum approach for this application thus far. Therefore, any algorithm can only be considered more feasible relative to the others for certain specific conditions. The quantifiable assessments in this study can help future research to optimize the filter parameters in different environments. Additionally, the information will also help to choose the most appropriate algorithm to process ECG signals in the specific circumstance.

\section{Acknowledgements}

The authors would like to thank the National Science Council, Taiwan, for supporting this research under Contract No. NSC95-2221-E-035-003.

\section{References}

[1] Widrow B, Glover J, McCool JM, Kaunitz J, Williams CS, Hearn RH, Zeidler JR, Dong E, Goodlin R. Adaptive noise cancelling: Principles and applications. Proc. IEEE 1975;63:1692-1716.

[2] Ahlstrom ML, Tompkins WJ. Digital Filters for RealTime Signal Processing Using Microprocessors. IEEE Trans. Biomed. Eng. 1985;32 (9):708-713.

[3] Pei SC, Tseng CC. Adaptive IIR Notch Filter Based on Least Mean p-Power Error Criterion. IEEE Trans. on Circuits and Systems-II: Analog and Digital Signal Processing 1993;41 No 8: 525-528.

[4] So HC. Adaptive algorithm for sinusoidal interference cancellation. Electron. Lett 1997;33 No. 22:1910-1912.

[5] Ziarani AK, Konrad A. A Nonlinear Adaptive Method of Elimination of Power Line Interference in ECG Signals. IEEE Trans. Biomed. Eng. 2002;49 No.6:540-547.

[6] Glover, Jr., J. R., "Comments on "Digital Filters for RealTime Signal Processing Using Microprocessors"” IEEE Trans. Biomed. Eng., Vol. BME-34 , No.12,pp. 962-963, December 1987

\section{Address for correspondence}

\author{
Name: Yue-Der Lin \\ Full postal address: Department of Automatic Control \\ Engineering, Feng Chia University, No.100 Wenhwa Rd., \\ Taichung City, 40724 Taiwan \\ E-mail address: ydlin@fcu.edu.tw
}

